\title{
ESTUDIO DE CLONALIDAD DE CEPAS DE KLEBSIELLA PNEUMONIAE MULTIDROGORESISTENTES CIRCULANTES EN EL INSTITUTO NACIONAL MATERNO PERINATAL DE LIMA, PERÚ (2015-2018)
}

\author{
CLONALITY OF MULTIDROGORESISTANTS KLEBSIELLA PNEUMONIAE \\ CIRCULATING AT THE NATIONAL MATERNAL AND PERINATAL INSTITUTE \\ OF LIMA, PERU (2015-2018)
}

\author{
Gabriela Soza Bieli-Bianchi ${ }^{1, a}$, Hortencia Acuña Fernández ${ }^{1, b}$, María Jesús Pons ${ }^{c}$
}

\begin{abstract}
RESUMEN
Objetivos: Determinar los niveles de resistencias a antimicrobianos y su relación de clonal en cepas de Klebsiella pneumoniae que presentan fenotipo multiresistente circulantes en el Instituto Materno- Perinatal de Lima, Perú. Materiales y métodos: el presente estudio es transversal que comprende a las cepas de K. pneumoniae aisladas durante el período 2015-2018. Se analizó los niveles de sensibilidad a los antimicrobianos mediante la técnica de difusión en Disco. Se estableció la relación clonal por la técnica de Gel de Elecroforesis de Campo Pulsado (PFGE de sus siglas en ingles). Resultados: Se analizaron un total de 36 aislados de K. pneumoniae de diferentes orígenes clínicos: un 23(64\%) hemocultivos, 9 $(25 \%)$ de secreciones y $4(11 \%)$ de coprocultivos. Se encontraron niveles elevados de resistencia a los antimicrobianos en cotrimoxazole (97\%), cefalosporinas (entre 67\%-89\%) y gentamicina (67\%). Destacar que la sensibilidad disminuida a quinolonas (36-47\%), y Piperacillin-tazobactam, nitrofurantoina y cloranfenicol (25\%), mientras que Amikacina presentó solo un $14 \%$ de resistencia. Un $78 \%$ de las cepas fueron cepas productoras de beta-lactamasas de espectro extendido (BLEE). EI PFGE mostró 16 pulsotipos diferentes, mostrando cepas relacionadas en el mismo espacio y tiempo. Conclusiones: Los niveles de resistencia encontrados fueron elevados, y destacar el número de cepas relacionadas clonalmente, evidenciando una diseminación de K.pneumoniae entre diferentes pacientes.
\end{abstract}

Palabras clave: Resistencia antibacteriana, clonalidad, Klebsiella pneumoniae (Fuente: DeCS BIREME).

\begin{abstract}
Objectives: To determine the antibiotic susceptibility and clonality relationship in Klebsiella pneumoniae strains that present multiresistance circulating in the Maternal-Perinatal Institute of Lima, Peru. Materials and methods: the present study is transversal and included K.pneumoniae strains isolated during the period 2015-2018. Antimicrobial susceptibility levels were analyzed using the diffusion technique.. The clonal relationship was established by the technique of Pulsed Field Electrophoresis Gel (PFGE). Results: A total of 36 K. pneumoniae isolates from different clinical origins were analyzed, 23 (64\%) blood cultures, $9(25 \%)$ secretions and $4(11 \%)$ stool samples. High levels of antimicrobial resistance were found in cotrimoxazole $(97 \%)$, cephalosporin $(67 \%-89 \%)$ and gentamicin $(67 \%)$. It should be noted that decreased susceptibility is important in quinolones (36-47\%), and moderate to Piperacillin-tazobactam, also in nitrofurantoin and chloramphenicol $(25 \%)$, while Amikacin presented only $14 \%$ resistance. A $78 \%$ of the strains were extended spectrum beta-lactamase (ESBL) producing strains. PFGE showed 16 different pulses, showing related strains in the same space and time. Conclusions: The levels of resistance found were high, and the number of related strains was also important, showing a dissemination of K.pneumoniae between different patients.
\end{abstract}

Keywords: Antibiotic resistance, clonality, Klebsiella pneumoniae (Source: MeSH NLM).

\section{INTRODUCCIÓN}

La resistencia a antimicrobianos es considerada un problema de salud global prioritario. Esto es especialmente relevante en los hospitales $y$, especialmente, en las Unidades de Cuidados Intensivos ( $\mathrm{UCl}$ ), donde debido al estado vulnerable de los pacientes, las adquisiciones de una infección asociada a atenciones hospitalarias
(HAI) son frecuentes y cada día más difíciles de tratar. Estas infecciones suelen ser causadas por patógenos especialmente adaptados a estos ambientes ${ }^{1}$, y se asocian al riesgo de transmisión cruzada entre pacientes o personal sanitario, dificultando su control ${ }^{2}$.

Klebsiella pneumoniae multidrogo resistente (MDR) es un patógeno bacteriano importante, responsable de brotes 
hospitalarios en todo el mundo, principalmente a través de la propagación de clones de alto riesgo ${ }^{3,4}$. La resistencia a antibacterianos en $K$. pneumoniae se ha relacionado con la presencia de beta-lactamasas de espectro extendido (BLEE), y, recientemente, con adquisición de carbapenemasas que confiere resistencia a los carbapenems y reduce de manera importante las opciones terapéuticas, e incrementa los costos relacionados ${ }^{5,6}$. La aparición y expansión global de clones de K. pneumoniae MDR e hipervirulentos se han reportado cada vez más a nivel mundial, tanto en infecciones adquiridas en la comunidad como en nosocomiales ${ }^{7,8}$.

La investigación y control de la resistencia antibiótica es clave en la salud global, sobre todo en los países de bajos y medianos ingresos, donde la prevalencia de cepas con resistencia a antimicrobianos es incluso mayor que en los países más industrializados ${ }^{9}$. De ahí que la propagación de la resistencia no es ajena a Perú, donde la carga de enfermedades infecciosas es elevada y los niveles de resistencia a antimicrobianos han alcanzado niveles preocupantes, principalmente en los patógenos más comúnmente aislados en centros de atención de la salud, con especial atención en las Unidades de Cuidados Intensivos ${ }^{10}$

A pesar de que el Perú presenta tasas de resistencia a los antimicrobianos más elevadas de la región ${ }^{11}$, los estudios de clonalidad de cepas MDR en los centros de salud son bien escasos. Este estudio permite una mejor comprensión de las estrategias preventivas y control de las infecciones en los centros de atención a la salud. Establecer la clonalidad de las cepas patógenas mediante la electroforesis de campo pulsado (PFGE, de sus siglas en inglés), es el método altamente discriminatorio y es excelente para tipificar bacterias e identificar si están relacionadas epidemiológicamente y podrían ser un brote ${ }^{12}$.

El objetivo del presente estudio fue establecer los niveles de sensibilidad a antimicrobianos y su relación clonal en aislados de K. pneumoniae MDR obtenidos de muestras clínicas (bacteremia, secreciones y coprocultivos) del Instituto Nacional Materno-Perinatal de Lima (período 2015-2018).

\section{MATERIALES Y MÉTODOS}

Diseño del estudio: Estudio descriptivo de tipo transversal en el cual se analizaron las cepas aisladas $(K$. pneumoniae) en las muestras biológicas (sangre, líquido cefalorraquídeo, aspirado bronquial, coprocultivos) obtenidas en los pacientes admitidos en el Instituto Nacional Materno Perinatal durante el período 20152018 .

Población y muestra: Se incluyeron en el estudio un total de 36 aislamientos clínicos de K. pneumoniae de diferentes pacientes. Estos aislados fueron seleccionados de una colección de bacterias del Laboratorio de Microbiología del Instituto Nacional Materno Perinatal de Lima. Sólo se incluyeron los aislados que presentasen un perfil de sensibilidad MDR (Resistencia a un antimicrobiano de al menos 3 familias de antibióticos diferentes $)^{13}$ mediante pruebas de difusión en disco, y que fueron conservadas y se pudieron revivir a la hora de realizar el estudio. Estos aislados fueron recolectados en el período comprendido entre 2015 y 2018.

Evaluación perfil sensibilidad a los antimicrobianos y fenotipo BLEE: Se procedió a realizar el antibiograma mediante la técnica de difusión en disco. La interpretación de los puntos de corte se realizó con la guía del CLSI (Clinical \& Laboratory Standards Institute del año 2019). Se realizó la evaluación de la presencia del fenotipo BLEE mediante el test de sinergia de doble disco ${ }^{14}$

Evaluación Clonalidad: Para definir la clonalidad entre las cepas de Klebsiella recolectadas se utilizó la técnica de la electroforesis de campo pulsado (PFGE) $)^{13}$. Brevemente, se digirieron los genomas bacterianos con el enzima de restricción Xbal. Se colocaron en el gel para electroforesis bajo las condiciones $(6,0 \mathrm{~V} /$ $\mathrm{cm}$ con un tiempo inicial de $5 \mathrm{~s}$ a un tiempo final de $35 \mathrm{~s}$ a $14{ }^{\circ} \mathrm{C}$ ) durante $24 \mathrm{~h}$. Se analizaron los perfiles para el análisis de los diferentes clones presentes ${ }^{13}$. Aquellos aislados que presentaron patrones de PFGE con un nivel de identidad del $100 \%$ se clasificaron como pertenecientes a un mismo clon y las que presenten una identidad $>80 \%$ serán consideradas como clonalmente relacionadas ${ }^{13}$.

Análisis de los resultados: En el presente estudio se realizó un análisis descriptivo de los resultados obtenidos, resumiendo las variables cualitativas según su frecuencia relativa y frecuencia absoluta. Para la relación clonal, se procedió a relacionar la filogenia según comparación de los patrones obtenidos en el PFGE. Así, los perfiles PFGE se compararon con el software InfoQuestTM FP v.4.5 (Bio-Rad, Hercules, CA). Con patrones electroforéticos, los dendrogramas se han construido utilizando el coeficiente de Dice, con agrupación por el método de grupos de pares no ponderados con media aritmética (UPGMA) con una tolerancia del $1 \%$ en las diferencias de posición de la banda.

Aspectos éticos: El presente estudio, teniendo en cuenta las buenas prácticas de investigación, es de riesgo mínimo. Toda la información clínica relevante o personal de los pacientes ha sido levantada de forma anónima, re-identificando con códigos alfa-numéricos. Todas las cepas de este estudio provienen de proyectos aprobados por el comité de ética de la Universidad científica del Sur y el del Instituto Nacional Materno Perinatal de Lima. 


\section{RESULTADOS}

Características generales. Se analizaron un total de 36 aislados de K.pneumoniae de diferentes orígenes clínicos, incluyendo 23 (64\%) aisladas de hemocultivos, 9 (25\%) de secreciones y $4(11 \%)$ de coprocultivos.

Perfil de sensibilidad a los antimicrobianos. De los 36 aislados testados por la técnica de difusión de disco en agar se han obtenido los siguientes resultados: 35 (97\%) aislados presentaban sensibilidad disminuida a cotrimoxazol, 33 (91\%) a tigecyclina. Referente a los betalactámicos, se ha encontrado un $26(72 \%)$ a aztreonam, 23 $(64 \%)$ a ceftazidima y $32(89 \%)$ a cefotaxime. También se encontraron valores elevados de resistencia para 24 (67\%) a gentamicina y 20 (56\%) a amoxicilina/ácido clavulánico. Para el grupo de las quinolonas, se encontró 17 (47\%) aislados con sensibilidad disminuida a ciprofloxacino y $13(36 \%)$ cepas a levofloxacino. Los valores de resistencia fueron menores para piperacilin / tazobactam, cloranfenicol y nitrofurantoína, cada uno con 9 (25\%) de aislados resistentes. Mencionar que se empiezan a encontrar cepas con resistencia a los carbapenems, 8 $(22 \%)$ a meropenem y $14(39 \%)$ a imipenem. Finalmente, solo $5(14 \%)$ aislados fueron resistentes a amikacina. De los 36 aislados incluidos en el estudio, un $78 \%$ resultaron positivos frente a la presencia de BLEE (Figura 1).
Niveles de resistencia (\%)

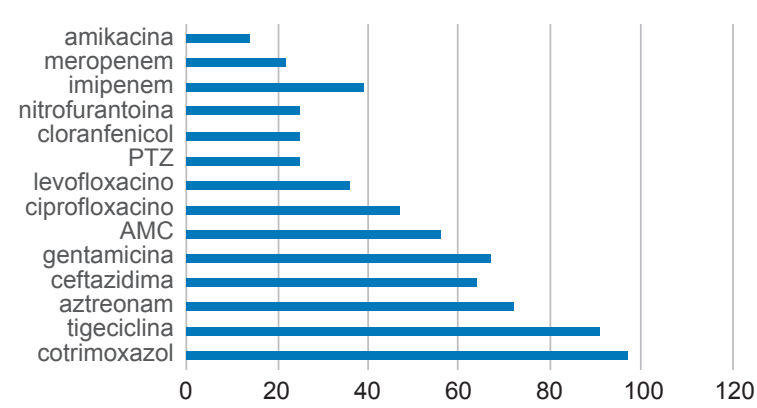

Figura 1. Porcentaje de resistencia a los antimicrobianos. PTZ: piperacillin/tazobactam. AMC: Amoxicilina/Ácido clavulánico.

Análisis de clonalidad por PFGE. Según el análisis de campo pulsado se detectaron 16 pulsotipos diferentes. Se destaca la presencia de cepas pertenecientes al mismo pulsotipo, incluyendo 7 del pulsotipo 7,5 del pulsotipo 16 , 4 del pulsotipo 14 y del 3,3 del pultotipo 12 y 2 de los pulsotipos 2 y 9 , respectivamente (Tabla 1). Distribución espacio temporal de los pulsotipos. Según el análisis de la distribución de los pulsotipos entre los diferentes servicios del INMP, se encontró que 3/7 aislados del pulsotipos 7 procedían del mismo clúster en tiempo y espacio, al igual que $3 / 5$ aislados del pulsotipo 16 , al igual que $2 / 4$ de los pulsotipos 3 y 14 .

Tabla 1. Dendograma y pulsotipos de la K.pneumoniae del estudio (2015-2018)

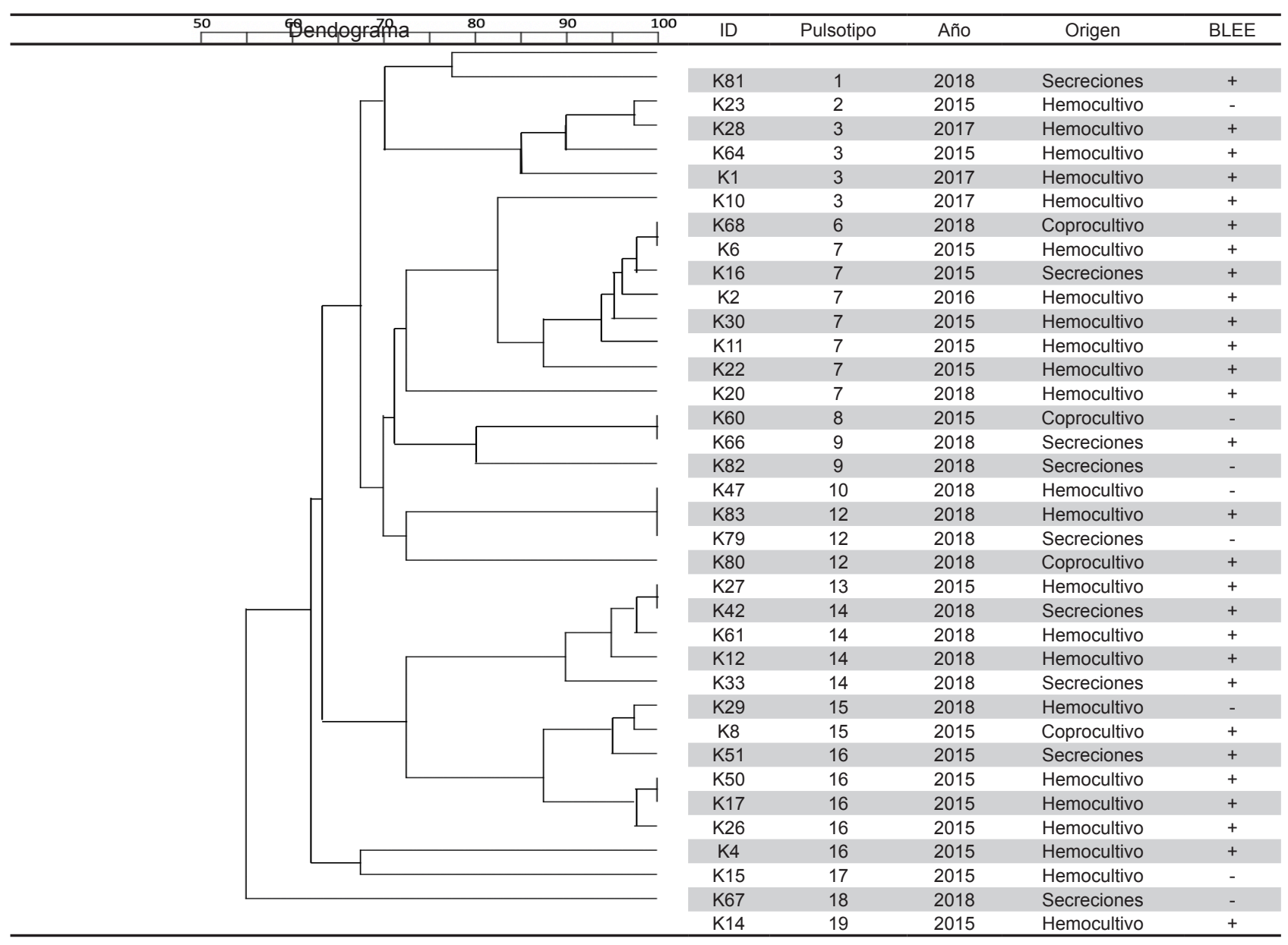




\section{DISCUSIÓN}

El presente estudio evidencia la presencia de cepas de $K$. pneumoniae MDR, especialmente destacando la presencia de BLEE, pertenecientes al mismo clon, o cepas clonalmente relacionadas circulando en el Instituto Nacional Materno Perinatal de Lima.

La presencia de brotes hospitalarios de Klebsiella productoras de BLEE, así como de otras Enterobacteriales en general, ha ido en aumento en América Latina ${ }^{15}$. Así, en Perú la frecuencia de bacteriemias producidas por Enterobacteriales productoras de BLEE adquiridas en la comunidad, sería la más alta reportada, comparado con otras regiones de Latinoamérica y Europa ${ }^{16}$. Dentro de las Enterobacterales, es Klebsiella spp. la que lidera la resistencia a la cefalosporina de tercera generación en la región, con valores reportados de $75 \%$ de resistencia ${ }^{17}$.

En este estudio se han reportado niveles elevados de resistencia a los antimicrobianos, sobre todo a cotrimoxazole, cefalosporinas y gentamicina. Se destaca que en estas cepas seleccionadas MDR, empiezan a aparecer la resistencia a carbapenems, que actualmente se está reportando como un problema emergente a nivel mundial ${ }^{18}$ y también en el Perú ${ }^{19}$. Una resistencia baja se ha reportado para amikacina (14\%), sugiriendo el rescate de algunos antimicrobianos más "antiguos", podría ser una opción antes de usar antibióticos de último recurso como colistina o las nuevas combinaciones de antimicrobianos ${ }^{20}$. El problema de la resistencia a los antibióticos en los países de ingresos medios-bajos tiene una especial relevancia debido a la debilidad de sus sistemas de salud, y, además, con frecuencia estos sistemas de salud no pueden permitirse el alto costo de los nuevos antimicrobianos para tratar los microorganismos extremadamente resistentes ${ }^{21,22}$. Este hecho hace que estos países sean particularmente vulnerables al aumento de la resistencia a los antibióticos ${ }^{21}$.

Observando la epidemiología, hemos encontrado varias cepas clonalmente relacionadas entre diferentes pacientes, sugiriendo una transmisión clonal de estas cepas. Se ha reportado la diseminación de patógenos entre pacientes, ya sea a través del contacto con familiares, personal médico o instrumentos contaminados, así, se ha reportado, como en nuestro estudio, una importante transmisión de patógenos de paciente a paciente productores de BLEE ${ }^{22}$. Relacionado con la transmisión, la especie de Klebsiella se ha reportado como más eficiente que otras especies como E.coli, tal vez relacionado con la importancia que está tomando a nivel de infecciones hospitalarias a nivel mundial ${ }^{23}$.

Diversos reportes evidencian la existencia de clones internacionales o epidémicos de $K$. pneumoniae en todo el mundo24; y algunas investigaciones han revelado mediante la genotipificación (MLST o secuenciación del genoma completo) la existencia de clones asociados con infecciones concretas ${ }^{24}$. Así es altamente preocupante la tendencia de $K$. pneumoniae a diseminarse en entornos hospitalarios, correlacionándose con la presencia de genes de resistencia de alta transmisibilidad ${ }^{18}$. Sin embargo, los estudios y caracterizaciones de la clonalidad en cepas circulantes en nuestro país son bien escaso, más allá de los brotes nosocomiales. Hacen falta más estudios de epidemiología molecular para la vigilancia de los principales patógenos resistentes a los antimicrobianos que circulan en nuestros centros sanitarios, a fin de poder obtener más conclusiones sobre la transmisión y evolución de las cepas de K. pneumoniae altamente resistentes a los antimicrobianos (especialmente carbapenems, colistina) en el Perú.

En conclusión se han visto varios clones y cepas relacionadas clonalmente en K.pneumoniae MDR del Instituto Materno Perinatal de Lima, revelando una transmisión de los patógenos. Se recomienda reforzar medidas de control para evitar la diseminación de microorganismos MDR en los centros de atención de salud.

Declaración de conflictos de intereses: Los autores declaran no tener conflicto de intereses.

Financiamiento: "Becas Iberoamérica Movilización. Jóvenes Profesores e Investigadores. Santander Universidades-2018" y "Fondos Semilla- Universidad Científica del Sur".

\section{REFERENCIAS BIBLIOGRÁFICAS}

1. Current HAl Progress Report. 2016 National and State Healthcare-Associated Infections Progress Report. https:// www.cdc.gov/hai/data/portal/progress- report.html.

2. Strich JR, Palmore TN. Preventing transmission of multidrugresistant pathogens in the intensive care unit. Infect Dis Clin North Am. 2017;31:535-50.

3. Palmeiro JK, de Souza RF, Schörner MA, Passarelli-Araujo H, Grazziotin AL, Vidal NM, Venancio TM, Dalla-Costa LM. Molecular Epidemiology of Multidrug-Resistant Klebsiella pneumoniae Isolates in a Brazilian Tertiary Hospital. Front Microbiol. 2019 Jul 23;10:1669.

4. Cubero M, Grau I, Tubau F, Pallarés R, Domínguez MÁ, Liñares J, Ardanuy C. Molecular Epidemiology of Klebsiella pneumoniae Strains Causing Bloodstream Infections in Adults. Microb Drug Resist. 2018 Sep;24(7):949-957.

5. Protonotariou E, Meletis G, Chatzopoulou F, Malousi A, Chatzidimitriou D, Skoura L Emergence of Klebsiella pneumoniae ST11 co-producing NDM-1 and OXA-48 carbapenemases in Greece. J Glob Antimicrob Resist. 2019 Sep 4;19:81-8.

6. European Centre for Disease Prevention and Control. Healthcare-associated infections acquired in intensive care units. In: ECDC. Annual epidemiological report for 2016. Stockholm: ECDC; 2018. (https://ecdc.europa.eu/sites/ portal/files/documents/AER_for_2016-HAI_0.pdf)

7. Girometti N, Lewis RE, Giannella M, et al. Klebsiella pneumoniae bloodstream infection: epidemiology and impact 
of inappropriate empirical therapy. Medicine (Baltimore). 2014;93(17):298-309.

8. Harada S, Aoki K, Yamamoto S, Ishii Y, Sekiya N, Kurai H, Doi Y. Clinical and molecular characteristics of Klebsiella pneumoniae causing bloodstream infections in Japan: occurrence of hypervirulent infections in healthcare. Journal of Clinical Microbiology. 2019 doi:10.1128/jcm.01206-19.

9. Laxminarayan R, Duse A, Wattal C, Zaidi AK, Wertheim HF, Sumpradit N, et al. Antibiotic resistance-the need for global solutions. Lancet Infect Dis. 2013;13:1057-98.

10. Center for Diseases Control and Prevention. Antibiotic resistance threats in the United States, 2013. Atlanta: US Department of Health and Human Services, CDC; 2013.

11. Garcia C. Antimicrobial resistance in Peru and Latin America. Acta méd. peruana v.29 n.2 Lima abr./jun. 2012.

12. Tenover FC, Arbeit RD, Goering RV et al. Interpreting chromosomal DNA restriction patterns produced by pulsedfield gel electrophoresis: criteria for bacterial strain typing. J Clin Microbiol 1995; 33: 2233-9.

13. Jiménez MA, Galas M, Corso A, Hormazábal JC, Duarte C,Salgado N, Ramón-Pardo P, Melano RG.Consenso latinoamericano para definir, categorizar y notificar patógenos multirresistentes, con resistencia extendida o panresistentesConsenso latinoamericano para definir, categorizar y notificar patógenos multirresistentes, con resistencia extendida o panresistentes.Consenso latinoamericano para definir, categorizar y notificar patógenos multirresistentes, con resistencia extendida o panresistentes Rev Panam Salud Pública. 2019; 43: e65.

14. Clinical and Laboratory Standards Institute (CLSI). Performance Standards for Antimicrobial Susceptibility Testing; Twenty-Seven Informational Supplement, M100-S28.

15. Sader H, Jones R, Andrade-Baiocchi S, Bie-denbach D. Fouryear evaluation of frequency of occurrence and antimicrobial susceptibility patterns of bacteria from bloodstream infections in Latin American medical centers. Diagn Microbiol Infect Dis. 2003;44:273-80. doi: 10.1016/S0732-8893(02)00469-8.

16. Sarmiento AF, Mejia MN, Rozas AB, Giraldo CA, Málaga G. [Frequency and risk factors for bacteremia caused by extended spectrum beta-lactamase-producing enterobacteriaceae in patients of a public hospital in Lima, Peru]. Rev Peru Med Exp Salud Publica. 2018 Jan-Mar;35(1):62-67. doi: 10.17843/ rpmesp.2018.351.3601.

17. Informe ReLAVRA, Revista de Patología Tropical V. 43, Supl. 2 - out./dez. 2014.

18. David S, Reuter S, Harris SR, Glasner C, et al. Epidemic of carbapenem-resistant Klebsiella pneumoniae in Europe is driven by nosocomial spread. a. Nat Microbiol. 2019 Jul 29.

19. Angles-Yanqui E, Huaringa-Marcelo J, SacsaquispeContreras R, Pampa-Espinoza L. Panorama de las carbapenemasas en Perú. Rev Panam Salud Publica. 2020;44:e61. https://doi.org/10.26633/RPSP.2020.61

20. Theuretzbacher U, Van Bambeke F, Cantón F et al. Reviving old antibiotics. Journal of Antimicrobial Chemotherapy. 201570 (8): 2177-2181.

21. Semret M, Haraoui LP. Antimicrobial Resistance in the Tropics Infect Dis Clin North Am. 2019;33(1):231-245.

22. Harris AD, Perencevich EN, Johnson JK, et al. Patient-topatient transmission is important in extended-spectrum betalactamase-producing Klebsiella pneumoniae acquisition. Clin Infect Dis. 2007;45:1347-50.

23. González ACR, Gil FG, Solórzano MR, et al. Outbreak of multiresistant and extended spectrum -lactamase producing Klebsiella pneumoniae in a high risk neonatal unit. Rev Chil Infectol. 2011;28:28-34.

24. Elhani D, Bakir L, Aouni M, et al. Molecular epidemiology of extended-spectrum b-lactamase- producing Klebsiella pneumoniae strains in a university hospital in Tunis, Tunisia, 1999-2005. Clin Microbiol Infect. 2010; 16: 157-164.

\section{Correspondencia:}

María Jesús Pons.

Dirección: Antig. Panamericana Sur, Km19. Lima, Perú

Teléfono: 943356470

E-mail: ma.pons.cas@gmail.com 